\title{
A Practically Linear Relation between Time Delay and the Optimal Settling Time of a Haptic Device
}

\author{
Thomas Hulin
}

\begin{abstract}
Haptic rendering denotes the process of computing and displaying forces from a virtual environment to a human operator via a haptic device. From the control point of view, the complete haptic system comprising virtual environment, haptic device, and human operator is a hybrid control system that contains both discrete- and continuous-time elements. This work investigates the influence of time delay on the theoretical optimal performance during haptic rendering with regard to minimal settling time, which belongs to the most frequently used design criteria in control engineering. It is shown that both continuous-time stiffness and damping of human operator or haptic device improve the optimal performance of the device. For the worst case, i.e., without such physical elements, the influence of delay on the optimal settling time becomes almost linear. This observation leads to an easy-to-remember rule of thumb for the optimal settling time that a haptic device should theoretically be able to reach. It states that each sampling period of additional time delay causes the optimal settling time (for a $2 \%$ position threshold) to increase by approximately five sampling periods. In this linear relation, the effect of discrete-time sampling appears to correspond to a delay of one whole sampling period. The theoretical investigations are accompanied by a series of experiments on a DLR/KUKA light-weight robot, which shows that the newly introduced rule of thumb also applies for single joints of complex robotic systems.
\end{abstract}

Index Terms-Haptics and Haptic Interfaces, Optimization and Optimal Control, Compliance and Impedance Control, Physical Human-Robot Interaction

\section{INTRODUCTION}

$\mathbf{C}$ OMPUTER-CONTROLLED mechanical systems involve both discrete- and continuous-time elements. Also haptic force feedback devices with digital controllers belong to this group of hybrid control systems. In haptic rendering, for instance, forces are computed from a virtual environment and displayed to a human operator via a haptic device [1]. The virtual environment and the controller of the haptic device are discrete-time elements. Discrete-time sampling, however, negatively affects the stability of a haptic system. For more than two decades, this effect is a topic of research in control engineering with the aims of (i) understanding the influence of time-discretization on stability and on parameter limitations of a haptic system, and (ii) finding suitable controllers that result stability under any condition.

A cornerstone in the control of haptic systems was established by Minsky et al. [2]. These authors performed two

Manuscript received: September 9, 2016; revised December 14, 2016; accepted February 22, 2017.

This paper was recommended for publication by Editor Yasuyoshi Yokokohji upon evaluation of the Associate Editor and Reviewers' comments.

Thomas Hulin is with the Institute of Robotics and Mechatronics, German Aerospace Center (DLR), D-82234 Wessling, Germany Thomas.Hulinddr. de

Digital Object Identifier (DOI): 10.1109/LRA.2017.2678538 analyses: an analytical continuous-time analysis that approximates the discrete-time effects by a delay, and a numerical simulation of a hybrid haptic system that contains discreteand continuous-time elements. This pioneering work motivated further extensive research from other researchers to investigate stability [3]-[7] or passivity as a tool for obtaining stability [8]-[12].

While the effect of discrete-time sampling on the stability of a haptic system was extensively investigated in the past and is well understood by now, there is still a clear gap of knowledge on how to optimally control a haptic device in order to achieve good performance. This is all the more remarkable since optimal control is a crucial topic for haptic devices. An ill-tuned haptic system may be stable but at the same time unsuited for any application, e.g., if slowly declining oscillations distort haptic sensation.

For the human user, an ideal haptic system would generate haptic feedback that creates the perfect illusion of an interaction with the real world. It would be able to recreate arbitrary force patterns, from contactless movements of the user's hand, over sliding along textured surfaces, to contacts with extremely rigid materials. In this context, Colgate and Brown [13] suggested to consider as a performance measure of haptic systems the size of the stable region, the so-called $Z$-width. However, although the Z-width is valuable to qualify the possible dynamic range of impedances of a haptic system, it cannot assess the actual dynamic performance of the system for given impedances. To this end, Salcudean and Vlaar [4] investigated the location of the system poles to determine optimal control parameters which settle quickly. Hulin et al. [14] enhanced this approach and considered the pole damping in addition to the settling behavior. Later, the same research group introduced performance measures to investigate the actual transient performance of a haptic device [15]. They also analyzed the influence of a human operator on the optimal performance of a haptic system. Paine and Sentis [16] recently investigated, in a completely different approach, the optimal performance of their system with regard to a phase margin criterion.

The present paper suggests using one of the most fundamental and best known design criteria in order to assess the performance of haptic devices: the settling time. This design criterion appears to be of particular relevance for haptic rendering, because even if the numerical value of contact stiffness is high, the appearance of long lasting oscillations may degrade the realistic impression of such stiff contacts with a virtual environment. The approach advances previous work [14], [15], [17], in which stability and optimal control design for haptic devices were investigated in the parameter 

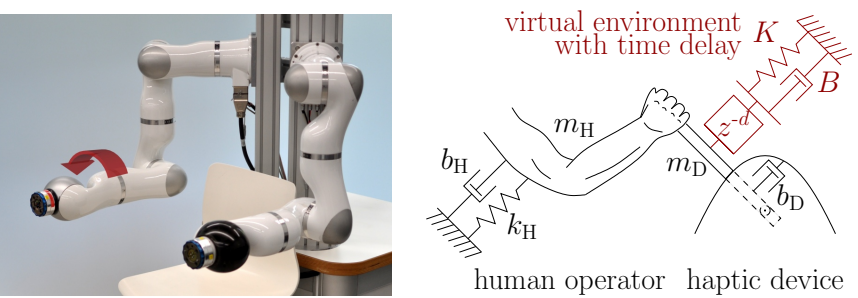

(a) Experimental setup with the (b) Linear model of a human arm interLight-Weight Robots of the bi- acting with a single degree of freedom manual haptic interaction device haptic device, which renders a discreteHUG [18]. The red round arrow time spring-damper system [17]. indicates the direction of the movements in the experiments.

Fig. 1. Experimental setup and considered model.

space. Sect. II describes the examined control system. Sect. III gives a brief summary of previous results on stability analysis of a haptic device. Sect. IV uses the optimal control approach introduced in [15] to investigate the theoretical control design based on the settling time. Sect. V analyses the influence of delay on these cost maps and finds a linear relation between delay and minimum settling time, which is formulated as an easy-to-remember rule of thumb. Sect. VI shows that this linear behavior can also be observed on a real complex device i.e. on a DLR/KUKA light-weight robot (see Fig. 1a). Sect. VII summarizes the results of this paper and discusses their implications.

\section{System Description}

This section briefly describes the considered system that is also shown in Fig. 1b. The reader is referred to [17] for details on all assumptions made and for a comprehensive discussion on the validity of the models.

The system consists of a human operator holding an impedance type haptic device, which interacts with a delayed virtual environment. The haptic device is a single degree of freedom (DoF) mass $m_{\mathrm{D}}$, which is damped by a viscous damper $b_{\mathrm{D}}$. It collides against a virtual wall that is represented by a discrete-time spring-damper system (discrete-time PDcontroller), with stiffness $K$, damping $B$, and constant sampling rate $1 / T$.

The total delay $t_{d}$ is considered as the sum of all delays involved in the closed-loop of the haptic device and the virtual world. This combined delay has a discrete-time transfer function of $z^{-d}$, where $d$ is the delay factor defined by $d=t_{d} / T$. In order to account for non-integer delay factor that may be evoked by asynchronous read and write operations or delays in the motor controller, the calculations in this paper admit rational numbers for the delay factor $d \in \mathbb{Q}_{\geq 0}$. This is made possible through a substitution of the discrete-time variable $z$, which results in an approximation of the transfer function that considers a discrete-time system with multiple of the original sampling rate.

The human arm is modeled as a single degree of freedom mass-spring-damper system with mass $m_{\mathrm{H}}$, stiffness $k_{\mathrm{H}}$ and viscous damping $b_{\mathrm{H}}$. It is further assumed that the human operator firmly holds the haptic device, such that the continuous-
TABLE I

NORMALIZATION RULES AND THE RESULTING DIMENSIONLESS PARAMETERS AS DEFINED IN [17].

\begin{tabular}{lcl}
\hline parameter name & symbol & dimensionless variable \\
\hline sampling period & $T$ & - \\
mass & $m$ & - \\
delay & $t_{d}$ & $d:=t_{d} / T$ \\
virtual stiffness & $K$ & $\alpha:=K T^{2} / m$ \\
virtual damping & $B$ & $\beta:=B T / m$ \\
physical stiffness & $k$ & $\gamma:=k T^{2} / m$ \\
physical damping & $b$ & $\delta:=b T / m$ \\
\hline
\end{tabular}

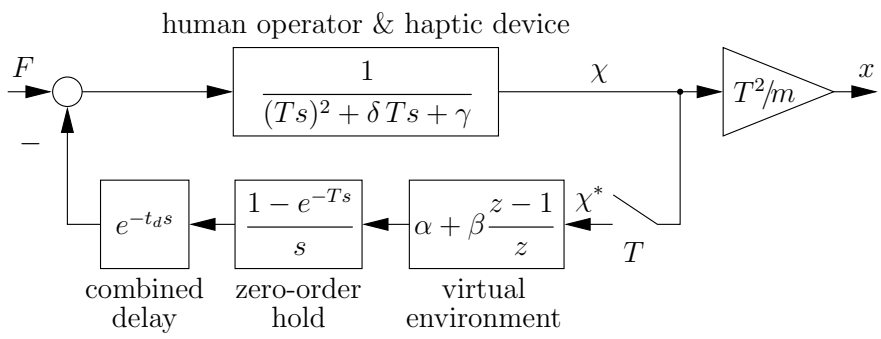

Fig. 2. Investigated control loop expressed by normalized parameters.

time parameters of haptic device and human may be combined to

$$
m=m_{\mathrm{D}}+m_{\mathrm{H}} \quad b=b_{\mathrm{D}}+b_{\mathrm{H}} \quad k=k_{\mathrm{H}},
$$

where $m, b$ and $k$ are the effective physical mass, damping and stiffness, respectively. The parameters of real haptic devices and human arms usually fulfill the two relations [17]:

$$
0 \leq k / m<1000 \mathrm{~s}^{-2} \quad 0 \leq b / m<20 \mathrm{~s}^{-1},
$$

where s means seconds, and should not be mixed up with the Laplace variable $s$.

\section{StABility ANALYSis IN A NutShell}

The optimal control design presented in this paper is based on a previous stability analysis [17], which investigates stability in the discrete-time domain. Hence, in order to fully understand the optimal control approach described in the present paper, it is necessary to briefly recap parts of previous work. In particular, this section introduces normalized system parameters and their realistic ranges and it discusses the effect of delay on the stable regions. The stability analysis in [17] is based on the location of the closed-loop system poles and consists of three main steps:

1) Calculate the discrete-time zero-order hold $(\mathrm{ZOH})$ equivalent transfer function of the continuous-time part of the system.

2) Introduce dimensionless substitution parameters.

3) Determine the stability boundaries in the plane of the normalized parameters.

The first step is necessary to analytically analyze the hybrid system, which consists of discrete- and continuous-time elements. It will not be discussed further here. However, it is important to note that this step is not an approximation but the resulting discrete-time system has the same behavior at the 


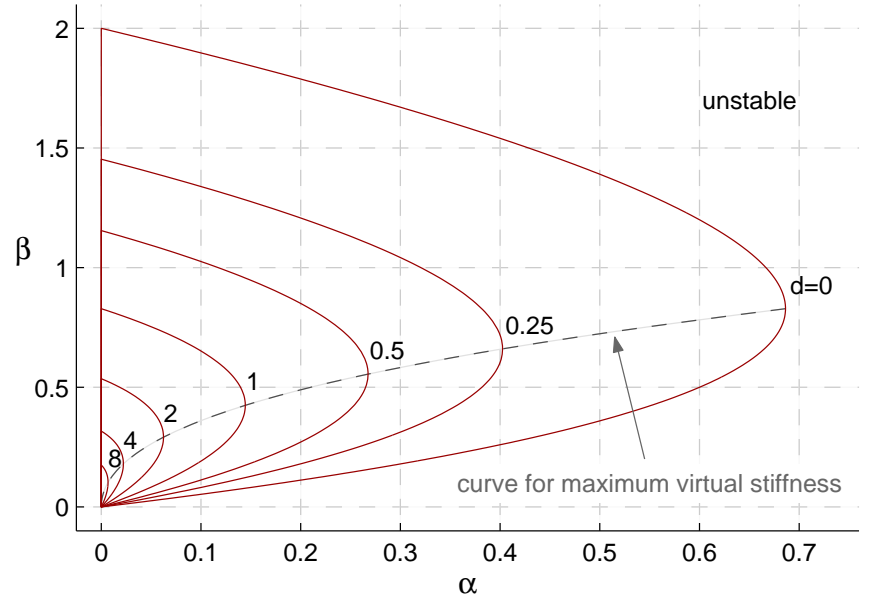

Fig. 3. Stability boundaries in the $(\alpha, \beta)$-plane for physical stiffness $\gamma=0$, physical damping $\delta=0$, and delay factors $d \in\{0,0.25,0.5,1,2,4,8\}$. The effect of delay $d$ is clearly visible as it shrinks the size of the stable regions.

sampling instants as the original hybrid system. The second step is important to understand the optimal control approach of the present paper, and hence it is described in detail in the following lines. Of the third step, only the resulting stability boundaries will be shown.

In the second step, the stiffness and damping parameters are substituted by their dimensionless counterparts as defined in Table I. These normalization rules result naturally by considering the characteristic equation of the system. The benefit of such substitution is threefold. Firstly, by applying these rules, the two parameters $m$ and $T$ drop out of the characteristic equation of the transfer function. Therefore, the original system, which contains seven parameters, is transformed into a system with only five explicit parameters. This simplified system only implicitly depends on $m$ and $T$. Fig. 2 shows the control loop, in which $\chi(t)$ is a scaled position and has the same unit of measurement as the input force $F$. Secondly, as the substitution parameters are dimensionless, the results hold for both translational and rotational movements. And, thirdly, the stability boundaries may be drawn in a normalized parameter plane, such that they hold independent of the mass $m$ and sampling period $T$.

Fig. 3 exemplarily shows the stability boundaries for the case $k=b=0$ (resp. $\gamma=\delta=0$ ) and illustrates the effect of additional time delay. The greater the delay factor $d$, the smaller becomes the stable region that is surrounded by the stability boundaries (red solid curves). The maximum stable stiffness $\alpha_{\max }$ is given by the rightmost point on the stability boundary. The dashed curve shows the course of this point in dependency of delay.

The effect of the two physical parameters of damping and stiffness is the following. An increase of physical damping $b$ (resp. $\delta$ ) leads to greater stable regions, whereas higher physical stiffness $k$ (resp. $\gamma$ ) mainly causes the stable region to move towards smaller virtual stiffness $K$ (resp. $\alpha$ ), i.e. leftwards in Fig. 3. However, for realistic values that are inside the parameters ranges given in (2), these parameters only have minor effect on the boundaries. In terms of the normalized parameters, these realistic parameter ranges can be determined

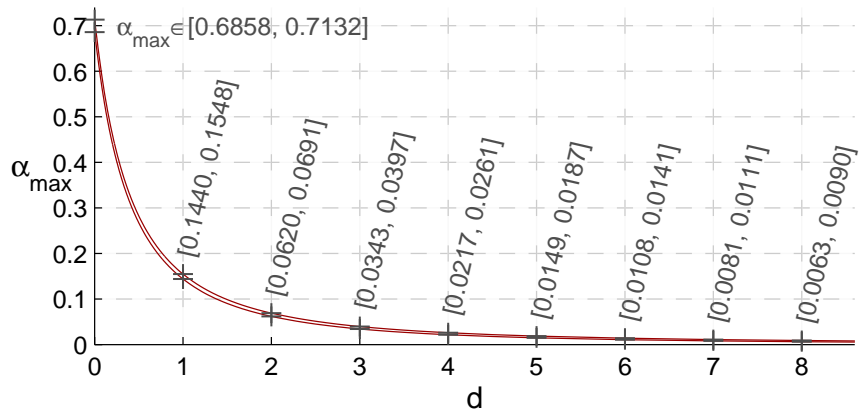

Fig. 4. Course of the maximum stable virtual stiffness $\alpha_{\max }$ over delay factor $d$ for the realistic parameter ranges in (2) resp. (3). The lower values result for $\gamma=0.001$ and $\delta=0$, and the upper values result for $\gamma=0$ and $\delta=0.02$.

by combining the limits of (2) with the widely accepted lower limit for the sampling rate of $1 \mathrm{kHz}[19]$, such that

$$
0 \leq \gamma<0.001 \quad 0 \leq \delta<0.02 .
$$

Fig. 4 shows the effect of these parameters on the maximum stiffness $\alpha_{\max }$. The numbers exemplarily give the value ranges in which $\alpha_{\max }$ varies at integer $d$ (the lower values result for $\gamma=0.001$ and $\delta=0$, the upper values result for $\gamma=0$ and $\delta=0.02$, the values for $\gamma=\delta=0$ are around 0.0005 greater than the lower values). It becomes obvious that for large delays of several sampling periods, the stability boundaries become small such that the relative effect of these two parameters becomes considerable. This figure also clearly shows how drastically the maximum stiffness $\alpha_{\max }$ decreases for an increasing delay.

\section{Theoretical Analysis of the Settling Time}

This section investigates the performance of the haptic device by means of the settling time. The settling time of a control system describes the duration of a unit system response until its output signal enters and stays inside a certain percentage threshold (also called the settling accuracy [20]) of its initial deflection. A common practice is to use $2 \%$ as percentage threshold value, and without loss of generality, the following discussion considers this threshold value. Later, the influence of the threshold value will be discussed by the example of two other common threshold values of $1 \%$ and $5 \%$.

The approach is similar to previous work [14] and [15], in which optimization criteria were investigated that consider the location of the system poles, the energy during the step response, or the overshoot. In accordance to that work, a dimensionless cost function for the settling time $T_{\text {settle }}$ is defined as

$$
C:=T_{\text {settle }} / T \text {. }
$$

It represents the number of sampling periods that the system takes to settle, which obviously is the same whether position $x$ or scaled position $\chi=x \cdot m / T^{2}$ (see Fig. 2) is considered. 


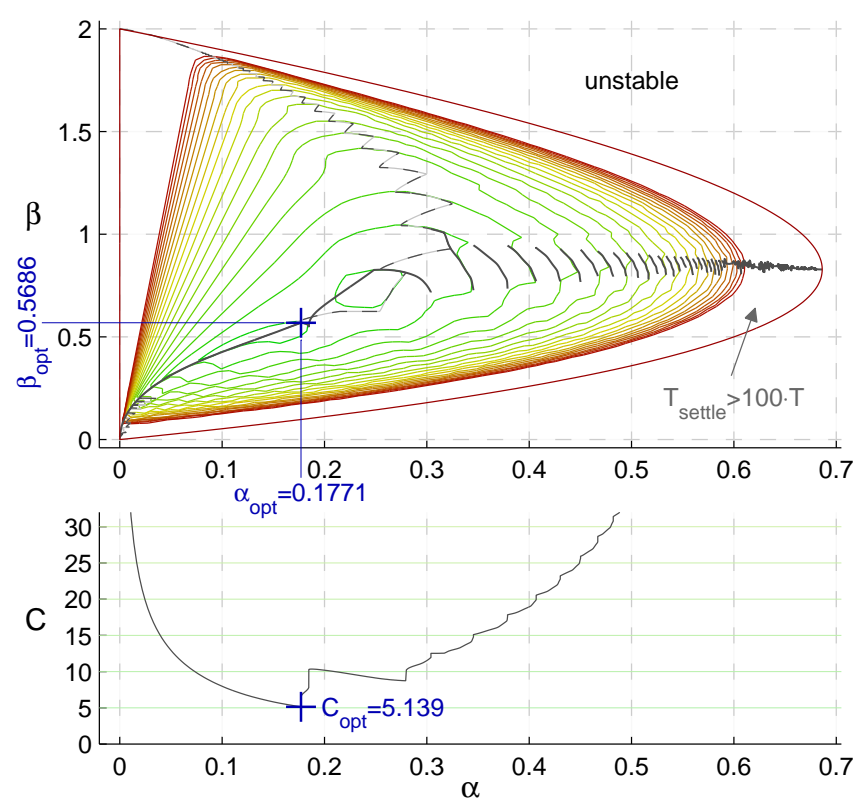

Fig. 5. Cost map for the settling time criterion visualized by contour lines for $\gamma=0, \delta=0$, and $d=0$. The lower subfigure is the front view on the cost map. The blue plus indicates the optimal point. The step response in this point is shown in Fig. 6.

\section{A. Cost Map}

In order to determine the costs inside the stable region, the stable region is gridded $^{1}$ and for each grid point the costs $C$ are determined using the unit step response of the system. This procedure results in a three-dimensional cost map that is visualized for practical purpose by contour lines of constant cost in the upper plot of Fig. 5 exemplarily for physical stiffness $\gamma=0$, physical damping $\delta=0$, and delay factor $d=0$. Between two contour lines, the costs $C$ differ by 5 . The cost distribution map has an absolute minimum value of $C_{\text {opt }}=5.139$. The corresponding optimum point is marked by a blue plus. It is notable that for a continuous-time system of second order without time delay, no such optimal point exists, but the settling time decreases with higher gains of stiffness and damping.

For each virtual stiffness $\alpha$ inside the stable region, there is a corresponding optimal damping value $\beta$, resulting in the black solid curve in Fig. 5. And, vice versa, for each virtual damping value $\beta$, there is an optimal virtual stiffness $\alpha$, resulting in the dashed curve. Note, the two optimal curves are identical for a certain range starting at the point of origin until they separate into two different curves. The lower subfigure shows the front view on the cost map and hence illustrates how the cost $C$ change when moving along the optimal curve for given $\alpha$ values (the solid curve).

The settling time of the considered haptic system in Fig. 1b is determined by the continuous-time position during a step response. However, in real haptic systems, the position is updated only at the sampling instants. This means that the costs that can be observed are discrete-time costs $C^{*}:=T_{\text {settle }}^{*} / T$

\footnotetext{
${ }^{1}$ All grids of the theoretical analysis in this paper have a resolution of 251 equally distributed grid points for each of the two axes, $\alpha$ and $\beta$, inside the bounding box of the stable region.
}

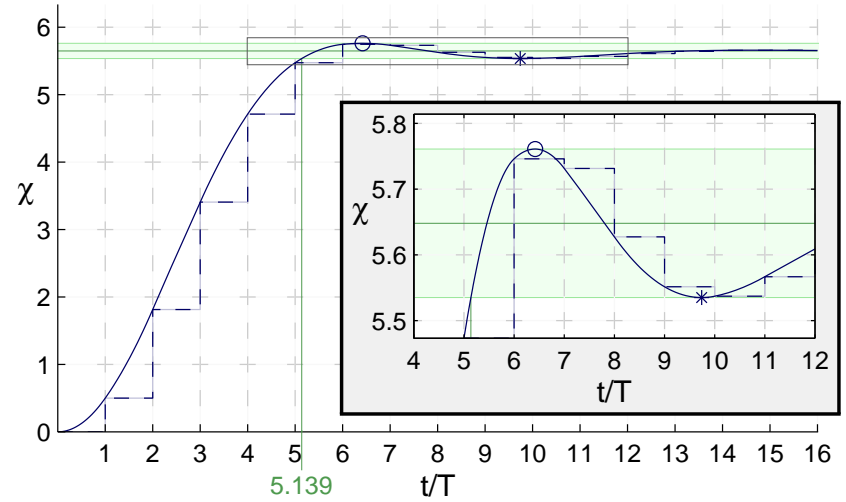

Fig. 6. The step response in the optimal point in Fig. 5 (i.e. for $d=0$, $\alpha=0.17705$, and $\beta=0.56865)$. The plot in the front shows a close-up view around final steady-state position $\chi_{\infty}=5.648 \mathrm{~ms}^{2} / \mathrm{kg}$. The points at which the response touches the $2 \%$ threshold band are marked by a circle and a star.

that can only take whole non-negative numbers $\mathbb{N}_{0}$. Although the discrete-time settling time $T_{\text {settle }}^{*}$ obviously differs from its accurate continuous-time counterpart $T_{\text {settle }}$, the resulting cost map looks quite similar to the one of the continuous-time cost $C$. An instance of a discrete-time cost map is shown in the experiments in Fig. 9.

\section{B. The Optimal Step Response}

In the optimal point, the settling cost with respect to the $2 \%$ settling time criterion becomes minimal. The continuous-time step response in this point is shown in Fig. 6. This figure also shows the discrete-time position $\chi^{*}$ as dashed line for the sake of completeness. After $T_{\text {settle, opt }}=5.139 T$, the continuoustime position $\chi$ stays inside the $2 \%$ threshold band (green shaded area). The plot in the front shows a close-up view around the final steady-state position $x_{\infty}=F_{0} /(K+k)$, respectively $\chi_{\infty}=F_{0} /(\alpha+\gamma)$, with $F_{0}=1 \mathrm{~N}$ because of the unit step. This plot reveals that the optimal step response touches the boundary of the $2 \%$ threshold band twice after entering it at $T_{\text {settle, opt }}$. First, it touches the upper boundary at its global maximum and then, at a local minimum, the lower one. In other words, in order to achieve the fastest settling time, the step response has to make use of the whole admissible threshold band. Therefore, if a threshold value other than $2 \%$ is taken into account, the optimal point would be located significantly beside the above optimum.

Table II shows the values of the optimal points for the three most common threshold values. These resulting values are given in normalized parameters and can be transformed into their non-normalized counterparts by the rules specified in Table I, i.e., for the virtual stiffness and damping of an optimal point, it holds that

$$
K_{\mathrm{opt}}=\alpha_{\mathrm{opt}} \cdot m / T^{2} \quad B_{\mathrm{opt}}=\beta_{\mathrm{opt}} \cdot m / T .
$$

Due to this parameter normalization, the results hold for arbitrary mass $m$ and sampling time $T$. For $d=0$, the effect of physical stiffness $\gamma$ and damping $\delta$ on the optimal cost (i.e. the cost in the optimal point) is small inside their realistic bounds. Additional physical damping $\delta$ slightly reduces the cost by less 
TABLE II

LOCATION AND COST OF THE OPTIMAL POINT FOR THE SETTLING TIME CRITERION WITH REGARD TO THREE DIFFERENT THRESHOLD VALUES FOR $\gamma=0, \delta=0$, AND $d=0$. THE LAST COLUMN GIVES THE SCALING FACTORS $\widetilde{C}(0)$ OF THE COST APPROXIMATION FUNCTION (6) DEFINED IN SECT. V.

\begin{tabular}{ccccc}
\hline threshold & $\alpha_{\text {opt }}$ & $\beta_{\text {opt }}$ & $C_{\text {opt }}$ & $\widetilde{C}(0)$ \\
\hline $1 \%$ & 0.1521 & 0.5304 & 6.159 & 5.812 \\
$2 \%$ & 0.1771 & 0.5686 & 5.139 & 4.896 \\
$5 \%$ & 0.2303 & 0.6462 & 3.876 & 3.772 \\
\hline
\end{tabular}

than $2 \%$, whereas the effect of physical stiffness $\gamma$ is even smaller (cost reduction of less than $0.5 \%$ ). As both physical parameters lead to a reduction of the optimal cost, the case without physical damping and stiffness, i.e. $\gamma=\delta=0$, may be considered as worst case in terms of optimal cost. The influence of delay is discussed in the following section.

\section{INFLUENCE OF DELAY}

The optimal points are highly affected by delay. As they are always located inside the stable region, they move with increasing delay towards the point of origin in a similar way to the stable regions. Even more revealing than the location of the optimal point is, however, how time delay affects the optimal cost of the haptic system. To clearly illustrate this effect, Fig. 7a shows the optimal cost for integer delay factors up to $d=8$. For each delay factor, the cost of the $1 \%, 2 \%$, and $5 \%$ thresholds are plotted as blue (circles), green (stars), and red (pluses) lines, respectively. For $\gamma=0$ and $\delta=0$, the cost for each threshold value seems to increase linearly with delay, yet with a different slope.

To investigate how accurately the costs follow such linear behavior, they are compared to a linear cost approximation function,

$$
\widetilde{C}(d):=\widetilde{C}(0) \cdot(d+1),
$$

where $\widetilde{C}(0)$ are the scaling factors or the slopes of the linear approximation. Their numerical values are listed in the rightmost column of Table II. To obtain more accurate values, they were determined for a delay factor of $d=32$ instead of $d=8$, although it can be checked that between these two delay factors, the relative difference is less than $0.07 \%$, i.e., the values only change in the last fractional digit shown. Fig. $7 \mathrm{~b}$ shows the relative error $\rho$ between the actual optimal costs and their linear approximation for $\gamma=0$ and $\delta=0$, with

$$
\rho:=\frac{C_{\mathrm{opt}}(d)-\widetilde{C}(d)}{C_{\mathrm{opt}}(d)} .
$$

The value of the relative error $\rho$ increases with decreasing delay, until it reaches its maximum at $d=0$. Or, in other words, the optimal performance of a haptic system with $\gamma=\delta=0$ approaches linear approximation (6) with increasing delay factor $d$. For $d=1$, the error is limited by $\rho \leq 1.5 \%$, while for the undelayed case $d=0$ it reaches $5.7 \%$ (for a $1 \%$ threshold).

On the other hand, in the previous section, it was observed that both physical stiffness $\gamma$ and damping $\delta$ have a positive effect on the optimal performance of a haptic system. This positive effect also appears for greater delay factors, even to a

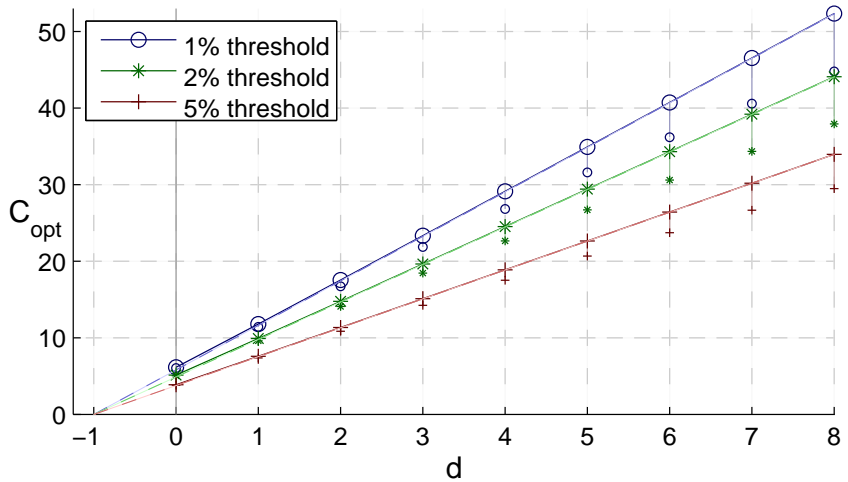

(a) optimal costs $C_{\text {opt }}$ (solid) and their linear approximations (dashed)

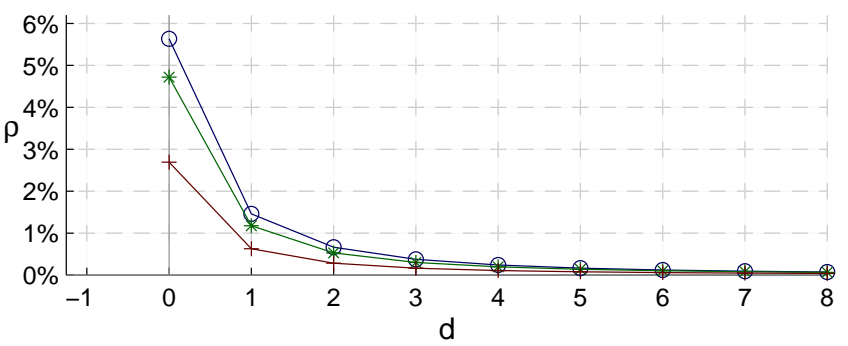

(b) relative error $\rho$

Fig. 7. Influence of delay $d$ on the costs $C_{\text {opt }}$ in the optimal points. The upper plot shows the costs for $\gamma=0$ and $\delta=0$ as solid curves, approximation function (6) as dashed lines, and the effect of physical stiffness $\gamma$ and physical damping $\delta$ up to their realistic limits as small markers below the respective curves. The lower plot shows the relative approximation error $\rho$ as defined in (7) for $\gamma=0$ and $\delta=0$.

higher extent than for $d=0$. Fig. 7a shows how the optimal cost reduces if these physical parameters are increased up to their realistic limits: the small markers below the respective curves represent the cost for $\gamma=0.001$ and $\delta=0.02$. If the two physical parameters $\gamma$ and $\delta$ are present, the influence of delay on the optimal performance becomes non-linear, but weaker than without these physical properties. Hence, linear approximation (6) may be considered as worst-case estimate for the optimal settling time. Or, to formulate it more clearly, if the virtual wall parameters $K$ (resp. $\alpha$ ) and $B$ (resp. $\beta$ ) are properly chosen, then real haptic devices should reach the performance specified by linear approximation (6). If some physical stiffness or damping is present, e.g. due to the human operator or the haptic device, then the device should even be able to outperform this performance value. Note, the relative error $\rho$ shown in Fig. 7b slightly degrades the validity of this statement especially for small delay factors.

Due to its fundamental importance, it is suggested to formulate linear approximation (6) as rules of thumb. For instance, for the $2 \%$ threshold, such a rule reads:

\section{Each sampling period of additional time delay causes the optimal settling time to increase by approximately five sampling periods.}

Another interesting property that can be learned from (6) is the effect of discrete-time sampling, which corresponds to a delay of one whole sampling period $T$ in terms of optimal cost. Its effect also becomes obvious in Fig. 7a, where linear approximation (6) crosses the horizontal axis at $d=-1$. This 
means that the cost would become zero at a theoretical delay of $t_{d}=-T$. Hence, in order to enable a desired performance, the total time period of the sum of all delays plus one whole sampling period is the decisive limiting factor, i.e.

$$
t_{\text {total }}:=t_{d}+T \text {. }
$$

For example, for the settling time criterion with a $2 \%$ tolerance band, this total time period must be less than approximately $20 \%$ of the targeted optimal settling time. In comparison, with respect to stability, the effect of discrete-time sampling corresponds to only half a sampling period delay (see e.g. linear stability condition in [17]).

\section{EXPERIMENTS WITH THE LIGHT-WEIGHT RoBOT}

This section presents experiments to support the validity of the theoretical approach but also to reveal limitations with respect to a real system. The bimanual haptic interaction device HUG [18], shown in Fig. 1a, was used as experimental platform. Deliberately, this device was chosen to show that some of the theoretical findings even hold for single joints of complex robotic systems, which differ from the considered model.

\section{A. Experimental Setup and Procedure}

HUG is equipped with two DLR/KUKA Light-Weight Robots (LWR) of the same type with similar dynamic behavior. These robot arms have a serial kinematics with seven joints that can be operated in torque and position control mode at an update rate of $1 \mathrm{kHz}$ [21]. They are controlled via sercos interface by a real-time computer, which means that jitter and package loss are negligible. The LWRs have a large workspace that is similar to that of a human arm.

An important difference to traditional haptic devices is that the LWRs are only poorly mechanically back-drivable. Instead, the LWR joints are equipped with torque sensors that allow for torque controlled operation mode with a controlled backdrivability. The respective controllers run on the joints' control electronics at a sampling rate of $3 \mathrm{kHz}$.

The experiments were conducted on the fifth joint of the left robot in Fig. 1a as indicated by the red round arrow. Choosing this joint is a tradeoff, as the joints with higher inertia (the ones that are closer to the base) excite stronger vibrations in the robot pedestal, whereas the joints with lower inertia are more susceptible to unconsidered effects such as static friction. All the other joints were turned off during the experiments, i.e., their internal brakes were activated.

For the investigated robot joint, the stability boundary and the cost map for the $2 \%$ settling time were determined. To this end, a discrete-time spring-damper system with modifiable parameters $K_{\text {rot }}$ and $B_{\text {rot }}$ was implemented in the rotational direction of joint five with a sampling rate of $1 \mathrm{kHz}$. To obtain the stability boundary, the maximum stable stiffness gains were determined for a predefined set of damping factors $B_{\text {rot }} \in\{0,1,2, \ldots, 40\} \mathrm{Nms} / \mathrm{rad}$ (in a previous short experiment, it was found that stable behavior could only be achieved for damping factors smaller than $B_{\text {rot }}<40 \mathrm{Nms} / \mathrm{rad}$ ). For each damping value $B_{\text {rot }}$, the stiffness $K_{\text {rot }}$ was increased by a specified step size of $200 \mathrm{Nm} / \mathrm{rad}$ until the device became unstable. In order to refine the critical stiffness gain and to obtain more precise stability boundaries, the step size of $K_{\text {rot }}$ was decreased to $20 \%$ of the original value in a second iteration.

For each tested parameter pair of virtual stiffness $K_{\text {rot }}$ and damping $B_{\text {rot }}$, the active joint was excited by an input torque step of $\tau_{0}=15 \mathrm{Nm}$ as indicated by the red round arrow in Fig. 1a and the step response was recorded. From these responses, the cost maps were computed offline after the experiments were finished and then compared to the theoretical results. The steady-state position of the joint was chosen to be always at the same position in the center of the joint's motion range, i.e., at a joint angle of $\Theta_{\infty}=0$. With these values, the experiment comprised 1025 step responses and took around six minutes. In order to reveal the influence of delay, the experiment was repeated six times with additional delays of up to $6 \mathrm{~ms}$. For these experiments, fewer step responses were recorded as smaller stable regions resulted.

\section{B. System Parameter Estimation}

In order to plot the theoretical curves and compare them to the experimental results, the three parameters of inherent time delay $t_{d}$, angular mass $I$, and physical damping $b_{\text {rot }}$ were estimated first. The physical stiffness was assumed zero $k_{\text {rot }}=0$ as there was no human operator involved. The intrinsic closed-loop delay without artificial delay was found to be constant at $t_{d}=2 \mathrm{~ms}^{2}$ The physical damping $b_{\text {rot }}$ was less than $0.1 \mathrm{Nms} / \mathrm{rad}$ (the damping was cancelled by the joint controller) and hence is neglected in the following evaluation. The estimated inertia value $I$ was determined to be $I=0.19 \mathrm{~kg} \mathrm{~m}^{2}$ using a method that is based on the conservation of momentum

As all three parameters were estimated directly from the step responses, these values are the ones that are visible from the controller side and thus determinant for the stability and performance of the controlled system. They may vary from their real physical values, as it is the case for physical damping that is compensated by internal controllers. Recall that errors in the estimated values have no effect on the experimental results, but only on the theoretical ones. The angular mass $I$ scales the theoretical stable regions, whereas the other physical parameters affect both the theoretical boundaries and their costs.

\section{Stability Boundaries}

Figure 8 shows the theoretical (left) and experimental (right) stability boundaries for an artificial delay of up to $6 \mathrm{~ms}$, i.e. a total closed-loop delay of $t_{d}=8 \mathrm{~ms}$. Both kinds of stable regions shrink with increasing artificial delay. A clear difference appears in the size of the theoretical and the experimental stability curves. The experimental curves are substantially smaller than the theoretical ones. This holds especially true for the height of the experimental regions,

\footnotetext{
${ }^{2}$ In comparison to previous experiments on this device [15], the total closedloop delay could be reduced to $2 \mathrm{~ms}$ by profound modifications in our custom software framework.
} 


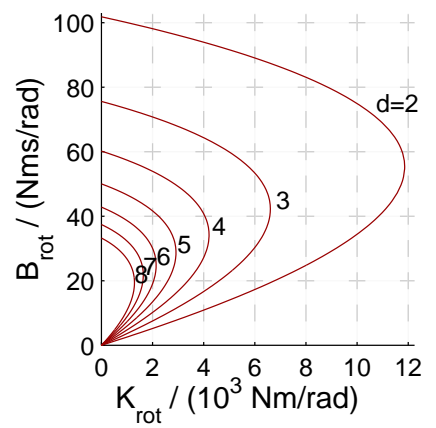

(a) theoretical boundaries

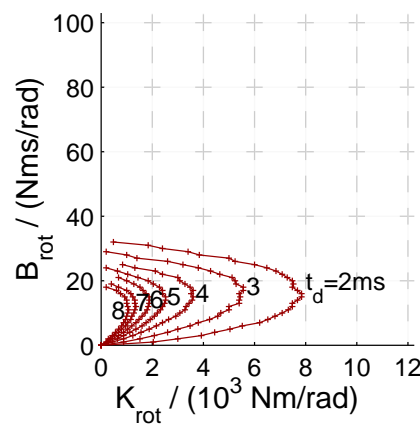

(b) experimental boundaries
Fig. 8. The influence of time delay on the theoretical (left) and on the experimental (right) stability boundaries. In the experiments, an artificial delay of up to $6 \mathrm{~ms}$ was introduced and increased at steps of $1 \mathrm{~ms}$. The theoretica curves (for $I=0.19 \mathrm{~kg} \mathrm{~m}^{2}, k_{\text {rot }}=0 \mathrm{Nm} / \mathrm{rad}$, and $b_{\text {rot }}=0 \mathrm{Nms} / \mathrm{rad}$ ) are shown for delay factors of $d \in\{2,3,4,5,6,7,8\}$ corresponding to a tota delay $t_{d}$ between $2 \mathrm{~ms}$ and $8 \mathrm{~ms}$. The plots show that with additional delay, both the experimental and the theoretical stable regions shrink.

which is between one third and one half of the theoretical regions' height.

By changing the estimated parameters, it would be possible to reach a better fit of the theoretical curves with the experimental ones (i.e., if a smaller inertia of $I=0.15 \mathrm{~kg} \mathrm{~m}^{2}$ is assumed). However, because the ratio of height to width is much lower for the experimental regions, which can not be compensated by varying the parameter estimates, a mismatch would remain. Hence, it can be concluded that parameter uncertainties are not the only reason for the observed discrepancy, but differences of the considered model to the real robot are also responsible for it. This is, however, not surprising taking into account that a robot that has some differences to the model was deliberately chosen as experimental platform. In particular, internal joint controllers, mechanical flexibility, and the non-linear effects of quantization, saturation and static friction are not considered in the conducted theoretical analysis and are potentially affecting stability.

\section{Cost Maps}

Figure 9 shows the theoretical and experimental cost maps for the experiment without additional delay for the $2 \%$ settling time. Note, the costs are discrete-time costs $C^{*}$ that are based on the discrete-time position signal. The shown theoretical plot assumes the parameter values estimated above $(d=2, I=$ $0.19 \mathrm{~kg} \mathrm{~m}^{2}, k_{\text {rot }}=0 \mathrm{Nm} / \mathrm{rad}$, and $\left.b_{\text {rot }}=0 \mathrm{Nms} / \mathrm{rad}\right)$. Similar to the form of presentation used in previous sections, these figures illustrate the cost distribution by contour lines. The distance between two lines corresponds to a change in the settling time of ten sampling periods. The optimal points of the cost maps are marked by blue plus signs.

Although the size of the stable regions is quite different between theory and experiments, there is some accordance in terms of cost. The contour lines indicate a similar evolution of cost inside the stable regions. More remarkable, however, is that the experimental optimal cost matches the theoretical findings: the optimal settling time is 15 sampling periods for both theory and experiment.
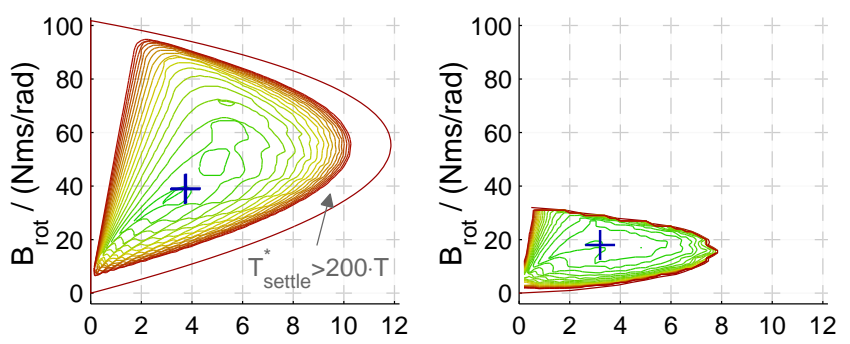

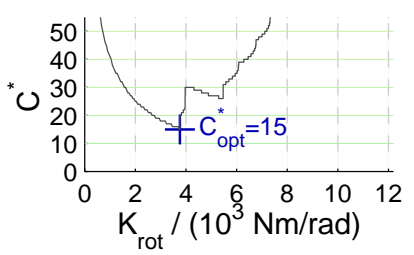

(a) theoretical cost map

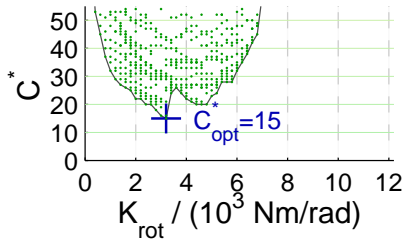

(b) experimental cost map
Fig. 9. Cost maps with contour lines and optimal curves for the $2 \%$ settling time assuming a delay of $d=2$. The theoretical cost maps (for $I=0.19 \mathrm{~kg} \mathrm{~m}^{2}, k_{\mathrm{rot}}=0 \mathrm{Nm} / \mathrm{rad}$, and $\left.b_{\mathrm{rot}}=0 \mathrm{Nms} / \mathrm{rad}\right)$ are on the left, and those of the LWR on the right. The optimal points are marked by blue plus signs. The lower subfigures are the front view on the cost map and hence show the minimal costs for given stiffness $K_{\text {rot }}$. Each green dot in the right plot represents the cost of one of the 1025 step responses.

\section{E. Influence of Delay on the Optimal Costs}

In order to reveal the influence of delay on the optimal costs, the cost maps for the $2 \%$ settling time were determined for each considered delay factor $d \in\{2,3,4,5,6,7,8\}$. Figure 10 compares the resulting optimal costs with the theoretical curves. The blue plus signs are the actual optimal costs that occurred during the experiments. The green solid lines represent the theoretical course of the optimal costs, i.e. the same curve as in Fig. 7a for a $2 \%$ threshold and for $\gamma=\delta=0$. The green dashed line shows the optimal costs for a physical damping of $\delta=0.02$ (resp. $\left.b_{\text {rot }}=3.8 \mathrm{Nms} / \mathrm{rad}\right)$, which is the upper limit of the realistic parameter range in (3). The green stars represent the theoretical discrete-time optimal costs $C_{\mathrm{opt}}^{*}$.

It can be clearly seen that the optimal settling time is prone to noise. In particular, for a delay factor of $d=7$, the experimentally determined optimal cost of $C_{\mathrm{opt}}^{*}=41$ is even slightly greater than its theoretical limit of 40 for $\gamma=\delta=0$. The reason for this discrepancy is, that the resolution of the experiment was not sufficient to find the actual optimal point for $d=7$ (in total only 81 stable step responses were recorded for this delay factor).

\section{CONCLUSiON}

Stability is a prerequisite for haptic rendering in which computed forces are displayed to a human operator. However, to generate effective and convincing haptic feedback, it is not sufficient to only consider stability. Rather, the haptic system has to achieve a certain performance. In this paper, it is suggested to use the settling time as a design criterion for haptic devices. Due to its practical usefulness and its simplicity, this criterion belongs to the most frequently used design criteria in control engineering.

For this criterion, three-dimensional cost maps were determined, which reveal the parameter pairs that result optimal 


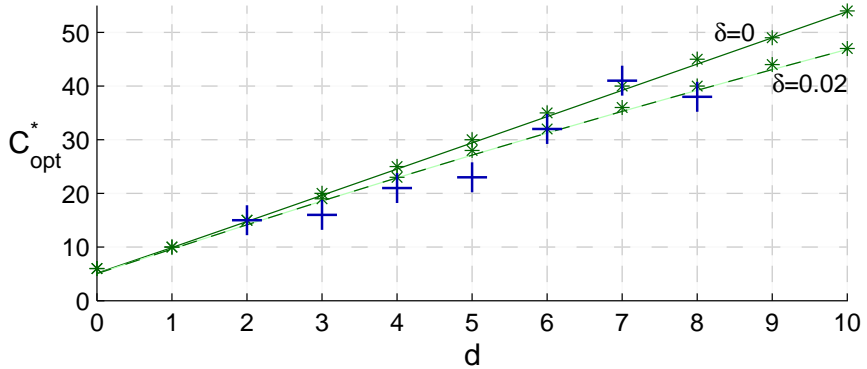

Fig. 10. The experimental versus the theoretical optimal costs $C_{\text {opt }}$ over time delay for the $2 \%$ settling time criterion. The optimal costs of the experiments are marked by blue plus signs for the robot. The green solid curves represent the course of the theoretical optimal costs for $\gamma=\delta=0$. The green dashed curves show the theoretical costs for a physical damping of $\delta=0.02$ $\left(b_{\text {rot }}=3.8 \mathrm{Nms} / \mathrm{rad}, k_{\text {rot }}=0 \mathrm{Nm} / \mathrm{rad}\right)$.

settling time. Since both continuous-time stiffness and damping improve this optimal performance, the worst-case situation is the one without these two physical parameters. For this situation, the influence of delay on the optimal cost becomes approximately linear. This linear relation represents a lower limit for the optimal performance of a haptic device, which means that any real haptic device with a minimal amount of physical damping should theoretically be able to outperform this limit. Due to this practical relevance, the linear relation was formulated as a rule of thumb. Interestingly, in terms of optimal cost, the effect of discrete-time sampling appears to correspond to a delay of one whole sampling period.

The optimal performance of a haptic system does not depend on its mass. The mass only proportionally scales the location of the optimal point, i.e. the optimal values of virtual stiffness and damping. In other words, two haptic devices with different masses but same sampling frequency and delay can theoretically achieve the same optimum settling time, given that both devices have the same normalized physical stiffness and damping gains. Experiments on a robot arm show that the rule of thumb even applies for systems that have some differences to the investigated control model. While the stability boundaries appeared to be sensitive to such modelling differences, the optimal performance could still be predicted well by the rule of thumb.

To conclude, the most important implication is that the approach allows for predicting the influence of the different system parameters on the optimal performance. This is of particular importance when designing new haptic devices or endeavoring to improve existing systems. However, the introduced approach may also serve as a tool to objectively assess novel controllers and to determine how much they outperform the traditional spring-damper implementation of a virtual environment.

Note that the investigated approach is valid only if the influence of other effects does not compromise the optimal performance of a haptic device. In certain cases, especially if the total time delay is small and the theoretical regions become large, the stability and the performance of the haptic system may be limited due to other factors including the nonlinear effect of sensor quantization [5], [12] or the mechanical flexibility of a haptic device [7]. Future research will tackle such interferences and will investigate other performance criteria as well as the human perception in the optimal points.

\section{REFERENCES}

[1] K. Salisbury, D. Brock, T. Massie, N. Swarup, and C. Zilles, "Haptic rendering: programming touch interaction with virtual objects," in SI3D '95: Symp. on Interactive $3 D$ graphics, Monterey, California, United States, 1995, pp. 123-130.

[2] M. Minsky, M. Ouh-young, O. Steele, F. Brooks Jr., and M. Behensky, "Feeling and seeing: Issues in force display," ACM SIGGRAPH Computer Graphics, vol. 24, no. 2, pp. 235-243, Mar. 1990.

[3] L. J. Love and W. J. Book, "Contact stability analysis of virtual walls," in Proc. of the ASME Dynamic Systems and Control Division, vol. 57. Georgia Institute of Technology, 1995, pp. 689-694.

[4] S. E. Salcudean and T. D. Vlaar, "On the emulation of stiff walls and static friction with a magnetically levitated input/output device," ASME Journal of Dynamic Systems, Measurement, and Control, vol. 119, no. 1, pp. 127-132, Mar. 1997.

[5] N. Diolaiti, G. Niemeyer, F. Barbagli, and J. K. Salisbury, "Stability of haptic rendering: Discretization, quantization, time delay, and coulomb effects," IEEE Trans. on Robotics, vol. 22, no. 2, pp. 256-268, Apr. 2006.

[6] R. Iskakov, A. Albu-Schäffer, M. Schedl, G. Hirzinger, and V. Lopota, "Influence of sensor quantization on the control performance of robotics actuators," in IEEE/RSJ Int. Conf. on Intelligent Robots and Systems (IROS), San Diego, CA, USA, Oct. 2007, pp. 1085-1092.

[7] P. Ciáurriz, I. Díaz, and J. J. Gil, "Stable discrete-time impedances for haptic systems with vibration modes and delay," IEEE Trans. on Control Systems Technology, vol. 22, no. 3, pp. 884-895, May 2014.

[8] J. E. Colgate and G. Schenkel, "Passivity of a class of sampled-data systems: Application to haptic interfaces," in 1994 American Control Conference. AIAA, 1994, pp. 37-47.

[9] R. J. Adams and B. Hannaford, "Stable haptic interaction with virtual environments," IEEE Trans. on Robotics and Automation, vol. 15, no. 3, pp. 465-474, Jun. 1999.

[10] B. E. Miller, J. E. Colgate, and R. A. Freeman, "Guaranteed stability of haptic systems with nonlinear virtual environments," IEEE Trans. on Robotics and Automation, vol. 16, no. 6, pp. 712-719, Dec. 2000.

[11] B. Hannaford and J.-H. Ryu, "Time-domain passivity control of haptic interfaces," IEEE Trans. on Robotics and Automation, vol. 18, no. 1, pp. 1-10, Feb. 2002.

[12] J. J. Abbott and A. M. Okamura, "Effects of position quantization and sampling rate on virtual-wall passivity," IEEE Trans. on Robotics, vol. 21 , no. 5, pp. 952-964, Oct. 2005.

[13] J. E. Colgate and J. M. Brown, "Factors affecting the z-width of a haptic display," in IEEE Int. Conf. on Robotics and Automation (ICRA), May 1994, pp. 3205-3210.

[14] T. Hulin, C. Preusche, and G. Hirzinger, "Stability boundary and design criteria for haptic rendering of virtual walls," in Int. IFAC Symp. on Robot Control (SYROCO), Bologna, Italy, Sep. 2006.

[15] T. Hulin, R. González Camarero, and A. Albu-Schäffer, "Optimal control for haptic rendering: Fast energy dissipation and minimum overshoot," in IEEE/RSJ Int. Conf. on Intelligent Robots and Systems (IROS), Tokyo, Japan, Nov. 2013, pp. 4505-4511.

[16] N. Paine and L. Sentis, "A closed-form solution for selecting maximum critically damped actuator impedance parameters," ASME Journal of Dynamic Systems, Measurement, and Control, vol. 137, no. 4, p. 041011, Apr. 2015.

[17] T. Hulin, A. Albu-Schäffer, and G. Hirzinger, "Passivity and stability boundaries for haptic systems with time delay," IEEE Trans. on Control Systems Technology, vol. 22, no. 4, pp. 1297-1309, Jul. 2014.

[18] T. Hulin, K. Hertkorn, P. Kremer, S. Schätzle, J. Artigas, M. Sagardia, F. Zacharias, and C. Preusche, "The DLR bimanual haptic device with optimized workspace," in IEEE Int. Conf. on Robotics and Automation (ICRA), Shanghai, China, May 2011, pp. 3441-3442.

[19] C. Basdogan and M. A. Srinivasan, "Haptic rendering in virtual environments," in Handbook of Virtual Environments: Design, Implementation, and Applications, K. Stanney, Ed. Erlbaum, 2002, pp. 117-134.

[20] M. E. Schlarmann and R. L. Geiger, "Relationship between amplifier settling time and pole-zero placements for second-order systems," in IEEE Midwest Symp. on Circuits and Systems, vol. 1, Lansing, MI, USA, Aug. 2000, pp. 54-59.

[21] G. Hirzinger, N. Sporer, M. Schedl, J. Butterfaß, and M. Grebenstein, "Torque-controlled lightweight arms and articulated hands: Do we reach technological limits now?" The Int. Journal of Robotics Research, vol. 23, no. 4-5, pp. 331-340, Jun. 2004. 